\title{
压力和酸响应的四苯乙烯修饰喹喔啉类荧光染料的合成与性质研究
}

\author{
孙静波张恭贺贾小宇薛鹏冲贾俊辉卢然* \\ (吉林大学化学学院 超分子结构与材料国家重点实验室 长春 130021)
}

\begin{abstract}
摘要 合成了新的给受体型四苯乙烯修饰的喹喔啉衍生物 BTPQ、DBTPQ 和 BTBQ. 三个化合物表现出不同程度的聚 集诱导发光(AIE)行为, 当 BTBQ (四苯乙烯单元修饰在喹喔啉的 2,3-位)的四氢呋喃溶液中含水量达到 $90 \%$ 时, 其荧光 发射强度增加至原来的 54 倍. 此外, 固体 BTBQ 在三氟乙酸蒸气作用下可由淡黄色变成红色，且其蓝绿色苂光被显著 猝灭, 可见, 它可作为传感材料用于酸蒸气的可视化检测. 由于连接在喹喔啉 5,8-位上的四苯乙烯单元的空阻作用导致 BTPQ 和 DBTPQ 不易被质子化，因此，二者对酸不敏感，但是，它们的固态发光颜色在研磨前后发生了明显变化，如， BTPQ 在结晶态时发射蓝色荧光, 经研磨变成无定形态后, 发射蓝绿色苂光, BTPQ 和 DBTPQ 的压致荧光变色行为在 研磨、加热/溶剂熏蒸处理下具有可逆性.

关键词 喹喔啉; 四苯乙烯; 压致荧光变色; 酸响应; 聚集诱导苂光
\end{abstract}

\section{Synthesis, Mechanochromism and Acid Response of the Fluorescence Dyes Based on Quinoxalines Modified with Tetraphenylethylenes}

\author{
Sun, Jingbo Zhang, Gonghe Jia, Xiaoyu Xue, Pengchong Jia, Junhui Lu, Ran* \\ (College of Chemistry, State Key Laboratory of Supramolecular Structure and Materials, Jilin University, \\ Changchun 130021)
}

\begin{abstract}
Three new D- $\pi$-A type quinoxalines modified with tetraphenylethylenes BTPQ, DBTPQ and BTBQ were synthesized via Suzuki coupling reactions between (4-(1,2,2-triphenylvinyl)phenyl)boronic acid and bromo aromatic hydrocarbons. It was found that BTPQ and DBTPQ, in which tetraphenylethylenes were substituted on 5,8-positions of quinoxalines gave the absortion bands at $316 \mathrm{~nm}$ and $303 \mathrm{~nm}$, respectively, originated from $\pi-\pi^{*}$ transition. For BTBQ, in which tetraphenylethylene units were located at 2,3-positions of quinoxaline, the $\pi-\pi^{*}$ transition absorption blue-shifted to $287 \mathrm{~nm}$ on account of the poor planarity and low conjugation. Meanwhile, the intermolecular charge transfer (ICT) emission could be detected for BTPQ and DBTPQ, whose emission bands red-shifted significantly and emission intensities decreased with increasing the solvent polarities. It should be noted that the three compounds exhibited aggregation-induced emission (AIE) behaviors. For instance, when the water faction in the THF solution increased to $90 \%$, the emission intensity at $c a$. $400 \mathrm{~nm}$ for BTBQ, was $c a .54$ times higher than that in THF. Additionally, trifluoroacetic acid (TFA) could lead to the changes of color and emitting color of BTBQ in solution as well as in solid state due to the formation of protonated quinoxaline. We found that the grey solid of BTBQ could turn into red one upon exposed to gaseous TFA, accompanying with the quench of the emission. Other kinds of acids of $\mathrm{HCl}, \mathrm{HNO}_{3}$ and acetic acid also could lead to the fluorescence quenching of solid BTBQ to some extent. Therefore, BTBQ could be used as sensory material to detect acid vapors by naked eyes. However, the protonation would be prohibited in BTPQ and DBTPQ on account of the steric effect of tetraphenylethylene units linked to 5,8-positions of quinoxaline, so BTPQ and DBTPQ could not detect acid. Interestingly, the solid emitting colors of BTPQ as well as DBTPQ were quite different before and after grinding, exhibiting mechanochromic properties. The as-prepared crystal of BTPQ emitting blue light under UV irradiation could be changed into amorphous powder with bluish green emission. The XRD patterns suggested that the mechanochromism was originated from the transition between the crystalline and amorphous states. Such mechanochromism was reversible under the treatment of grinding and heating/fuming with DCM.

Keywords quinoxaline; tetraphenylethylene; mechanochromism; acid response; AIE
\end{abstract}

\section{1 引言}

压致荧光变色材料是指其固态苂光发射行为在外 力(如压力、剪切力、拉伸力等)作用下能发生明显改变
的一类刺激响应性智能材料, 在应力传感、位移或形变 检测、防伪、记忆芯片、光学存储、光电器件和显示器 件等领域具有重要的应用价值 ${ }^{[1]}$. 一般认为, 外力诱导 下的分子构象的改变或分子堆积方式的改变是引起压

*E-mail: luran@mail.jlu.edu.cn

Received September 25, 2015; published October 27, 2015.

Supporting information for this article is available free of charge via the Internet at http://sioc-journal.cn.

Project supported by the National Natural Science Foundation of China (No. 21374041) and the Open Project of State Key Laboratory of Supramolecular Structure and Materials (No. SKLSSM2015014).

项目受国家自然科学基金(No. 21374041)和超分子结构与材料国家重点实验室开放课题(No. 2015014)资助. 
致苂光变色现象的重要原因 ${ }^{[2]}$. 另外, 值得一提的是, 自唐本忠教授首次提出聚集诱导发光 (Aggregationinduced emission, AIE) 这一概念以来 ${ }^{[3]}$, 人们对具有强 固态发光的材料给予了高度关注 ${ }^{[1 \mathrm{c}, 4]}$. 研究表明, AIE 分 子通常具有特殊的扭曲构象, 在溶液中的发光能力较 差, 但在固态时, 由于分子中围绕单键的自由旋转受到 抑制, 从而有利于辐射跃迁的发生, 显示出较强的荧光. 然而, 具有扭曲构象的分子在固相中通常难以紧密堆 积, 在外力作用下容易发生分子堆积方式的改变, 进而 产生压致变色现象. 因此, 人们发现某些 AIE 材料具有 压致荧光变色性质, 而非平面共轭单元(例如, 四苯乙 烯 ${ }^{[1 b, 5]}$ 、二苯乙烯基葱 ${ }^{[6]}$ 、三苯胺 ${ }^{[7]}$ 和三苯基丙烯腈 ${ }^{[8]}$ 等) 的引入成为设计压致荧光变色染料的策略之一. 至今, 人们已经制备了多种类型的具有压致荧光变色性质的 有机共轭分子. 如, Araki 和 Kato 发现基于苯基取代菲的 氢键自组装体能在外力作用下由发蓝色苂光的固体转 化成发绿色荧光的固体, 他们还报道了联葱类液晶材料 的压致荧光变色性质 ${ }^{[9]}$. 特别地, 由于 ICT (分子内电荷 转移)发射对外界微环境的变化比较敏感, 因此人们对 新型 D- $\pi-A$ 型苂光染料分子的设计产生了兴趣 ${ }^{[10]}$. Harima 报道了一系列具有压致苂光变色性质的给受体型 稠杂环化合物 ${ }^{[11]}$. 我们课题组也合成了以咔唑或三苯胺 为电子给体, 以苯并噁唑或 $\beta$-亚胺酮硼络合物为电子受 体的 D- $\pi-A$ 型压致荧光变色材料 ${ }^{[12]}$, 发现具有适当极性 的共轭分子的 ICT 跃迁发射带对外界压力显示出较显 著的刺激响应. 同时还发现将非平面的吩噻嗪修饰到 蒽、 $\beta$-亚胺酮嗍络合物以及三苯基丙烯腈衍生物上亦可 得到压致荧光变色染料 ${ }^{[13]}$, 且吩噻嗪在受体单元上的 不同连接方式可导致其压致荧光变色性质的差异. 本文 中, 考虑到喹喔啉是典型的电子受体, 且其 2,3,5,8-位易 于功能化, 故考虑将具有 AIE 活性的四苯乙烯单元修饰 到喹喔啉的不同位置, 合成新的喹喔啉衍生物 BTPQ, DBTPQ 和 BTBQ (Scheme 1), 以期获得固态发光可调 控的苂光染料, 揭示分子结构对其 AIE 和压致荧光变色 性能的影响. 另外, 由于喹喔啉的质子化可导致其发光 性质的改变 ${ }^{[14]}$, 因此, 具有较强固态发光能力的喹喔啉 衍生物有望作为苂光探针, 实现对酸蒸气的检测.

\section{2 结果与讨论}

\section{1 目标分子的合成}

4-(1,2,2-三苯乙烯基)-苯硼酸(1)分别与化合物 2、3 和 4 在 $\mathrm{Pd}\left(\mathrm{PPh}_{3}\right)_{4}$ 催化下、经 Suzuki 偶联反应得到目标 产物 BTPQ 和 DBTPQ 以及中间体 5, 产率分别为 75\%, $80 \%$ 和 $63 \%$. 化合物 5 与邻苯二胺在乙酸溶液经缩合成 环过程生成了 2,3-二芳基取代的 BTBQ, 产率为 95\%. 目标分子的结构经核磁共振氢谱、核磁共振碳谱、红外 光谱和质谱进行了表征.

\section{2 溶液中紫外-可见吸收和荧光发射光谱}

图 1 给出了喹喔㕲衍生物 BTPQ、DBTPQ 和 BTBQ 在二氯甲烷溶液 $\left(1.0 \times 10^{-5} \mathrm{~mol} \cdot \mathrm{L}^{-1}\right)$ 中的紫外-可见吸收 光谱. 可见, 在喹喔啉的 5,8-位连有四苯乙烯单元的 BTPQ 在 $316 \mathrm{~nm}$ 处给出了一个归属于 $\pi-\pi$ *跃迁的吸收 带，在 361 395 nm 之间给出了一个来源于 ICT 跃迁的 吸收带. 当在 BTPQ 的 2,3-位分别引入苯基后，化合物 DBTPQ 的 $\pi-\pi$ * 跃迁吸收峰蓝移至 $303 \mathrm{~nm}$, 同时, ICT 跃 迁吸收带明显变宽. 对于在喹喔啉的 2,3-位连有四苯乙 烯单元的 BTBQ 而言, 其 $\pi-\pi *$ 跃迁吸收峰蓝移至 287 $\mathrm{nm}$, 可能是由于在喹喔啉的 2,3-位引入体积较大的四苯 乙烯可降低分子的共平面性, 从而使其共轭程度下降.

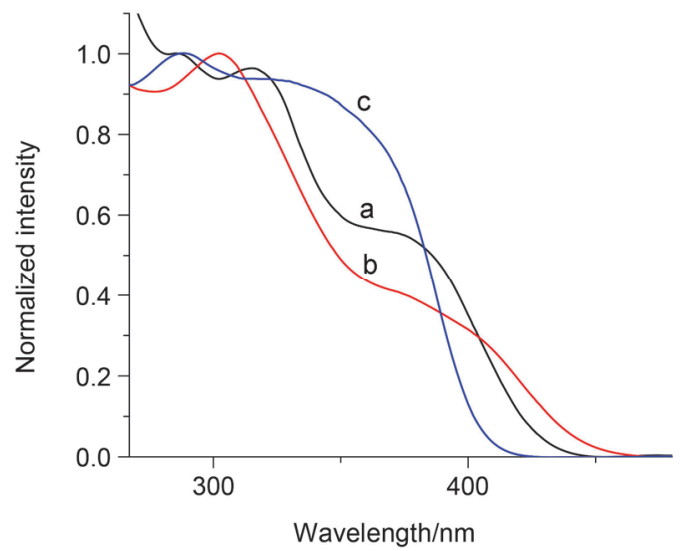

图 1 归一化的 BTPQ (a), DBTPQ (b)和 BTBQ (c)在二氯甲烷溶液 $\left(1.0 \times 10^{-5} \mathrm{~mol} \cdot \mathrm{L}^{-1}\right)$ 中的紫外-可见吸收光谱

Figure 1 Normalized UV-vis absorption spectra of BTPQ (a), DBTPQ (b) and BTBQ (c) in DCM $\left(1.0 \times 10^{-5} \mathrm{~mol} \cdot \mathrm{L}^{-1}\right)$

图 S1 给出了 BTPQ、DBTPQ 和 BTBQ 在不同溶 剂中的紫外-可见吸收光谱和荧光发射光谱, 相应的光 物理数据列于表 S1. 结果发现, 三个化合物的紫外-可 见吸收光谱随着溶剂极性的改变没有发生明显的变化. 但是，随着溶剂极性的增加, BTPQ 和 DBTPQ 的荧光发 射带逐渐红移, 且其荧光量子产率 $\left(\Phi_{\mathrm{F}}\right)$ 逐渐下降, 例如, 在正已烷中, DBTPQ 的最大发射峰出现在 $482 \mathrm{~nm}$, 发 射蓝色荧光(图 S2), $\Phi_{\mathrm{F}}$ 为 0.48 ; 在 DMF 中, DBTPQ 的 最大发射峰红移至 $533 \mathrm{~nm}$, 发射黄色苂光, $\Phi_{\mathrm{F}}$ 降为 0.14 . 故, 可以推断 BTPQ 和 DBTPQ 在极性溶剂中的苂光发 射来源于 ICT 跃迁 ${ }^{[13]}$. 但是, BTBQ 的苂光发射光谱并 没有随着溶剂极性的增加呈现出规律性的变化, 说明没 有给出典型的 ICT 发射峰.

\section{3 密度泛函理论计算}

为了进一步了解 BTPQ、DBTPQ 和 BTBQ 的电子 结构, 对其进行了密度泛函量化计算. 如图 2 所示, 化 合物 BTPQ 与 DBTPQ 的 HOMO 主要集中在四苯乙烯 单元及其相连的喹喔啉中的苯环上, 而 LUMO 在喹喔 啉核上的分布明显高于在四苯乙烯上的分布，说明在化 合物 BTPQ 和 DBTPQ 中有利于实现电荷从给体四苯乙 
<smiles>OB(O)c1ccc(C(=C(c2ccccc2)c2ccccc2)c2ccccc2)cc1</smiles>

1<smiles>OB(O)c1ccc(C(=C(c2ccccc2)c2ccccc2)c2ccccc2)cc1</smiles>

1<smiles>OB(O)c1ccc(C(=C(c2ccccc2)c2ccccc2)c2ccccc2)cc1</smiles>

1

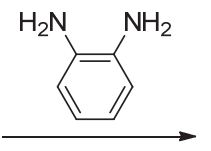

HOAc, reflux<smiles>Brc1ccc(Br)c2nccnc12</smiles>

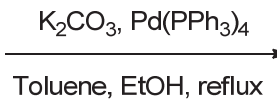

2<smiles>Brc1ccc(Br)c2nc(-c3ccccc3)c(-c3ccccc3)nc12</smiles>

3

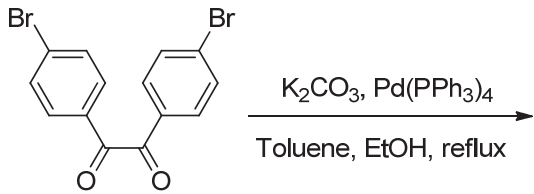

4<smiles>c1ccc(C(=C(c2ccccc2)c2ccc(-c3ccc(-c4ccc(C(=C(c5ccccc5)c5ccccc5)c5ccccc5)cc4)c4nccnc34)cc2)c2ccccc2)cc1</smiles>

BTPQ

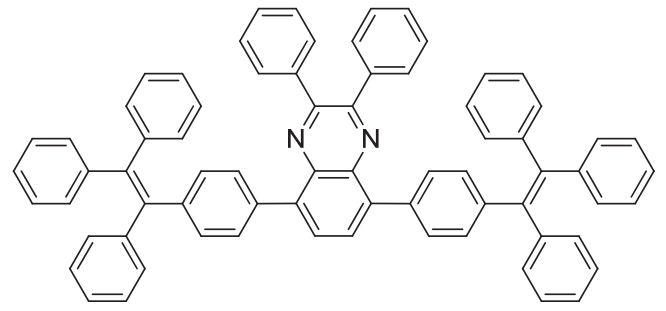

DBTPQ

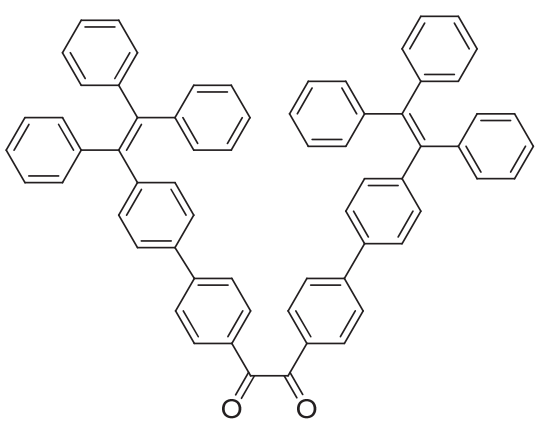

5

图式 1 BTPQ, DBTPQ 和 BTBQ 的合成路线

Scheme 1 Synthetic routes for BTPQ, DBTPQ and BTBQ

烯单元到受体喹喔啉和 2,3-二苯基喹喔啉上的转移 ${ }^{[15]}$, 即发生 ICT. 化合物 BTBQ 的 HOMO 和 LUMO 都主要 集中在四苯乙烯及其相连的苯环上, 在吡嗪环上的分布 很小, 这可能也是导致其不能给出典型的 ICT 发射的原 因.

\section{4 聚集诱导发光性质}

众所周知, 四苯乙烯是典型的具有 AIE 活性的结构 单元, 因此, 本文研究了所合成目标化合物的 AIE 性 质 ${ }^{[16]}$. 如图 3 所示, 当混合体系中水含量 $\left(f_{\mathrm{w}}\right)$ 低于 $80 \%$ 时, BTBQ 在水/THF 中的荧光发射非常弱; 当 $f_{\mathrm{w}}>80 \%$ 时, 其苂光发射强度明显增强, 当 $f_{\mathrm{w}}$ 为 $90 \%$ 时, 苂光发
射强度达到最大，是其四氢呋喃溶液中的 54 倍. BTPQ 和 DBTPQ 也表现出类似的 AIE 性质. 当混合体系中 $f_{\mathrm{w}} \leqslant 60 \%$ 时, BTPQ 和 DBTPQ 的苂光很弱，且其苂光发 射强度随着含水量的增加而下降(图 $\mathrm{S} 3 \sim \mathrm{S} 4$ ), 原因在 于: 随着含水量的增加, 溶剂的极性逐渐增大, 从而导 致其 ICT 发射强度逐渐下降; 当 $f_{\mathrm{w}}>60 \%$ 时，伴随着水 含量的增加, 分子的荧光发射逐渐增强; 当 $f_{\mathrm{w}}=90 \%$ 时, 苂光发射强度均达到最大, 分别是其四氢呋喃溶液中的 7.7 倍和 2.7 倍. 综上, 在喹喔啉的 2,3-位上修饰四苯乙 烯单元的分子给出最显著的 AIE 性质. 值得一提的是, 在固态下 BTBQ 能发射较强的浅绿色荧光, 固态荧光量 


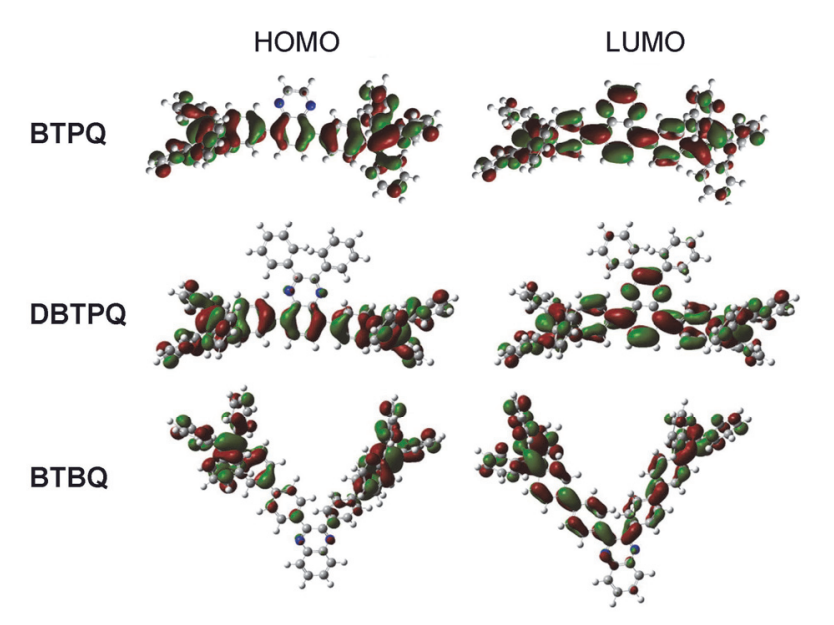

图 2 BTPQ, DBTPQ 和 BTBQ 的 HOMO 和 LUMO 前线轨道图 Figure 2 The frontier orbital plots of the HOMO and LUMO of BTPQ, DBTPQ and BTBQ
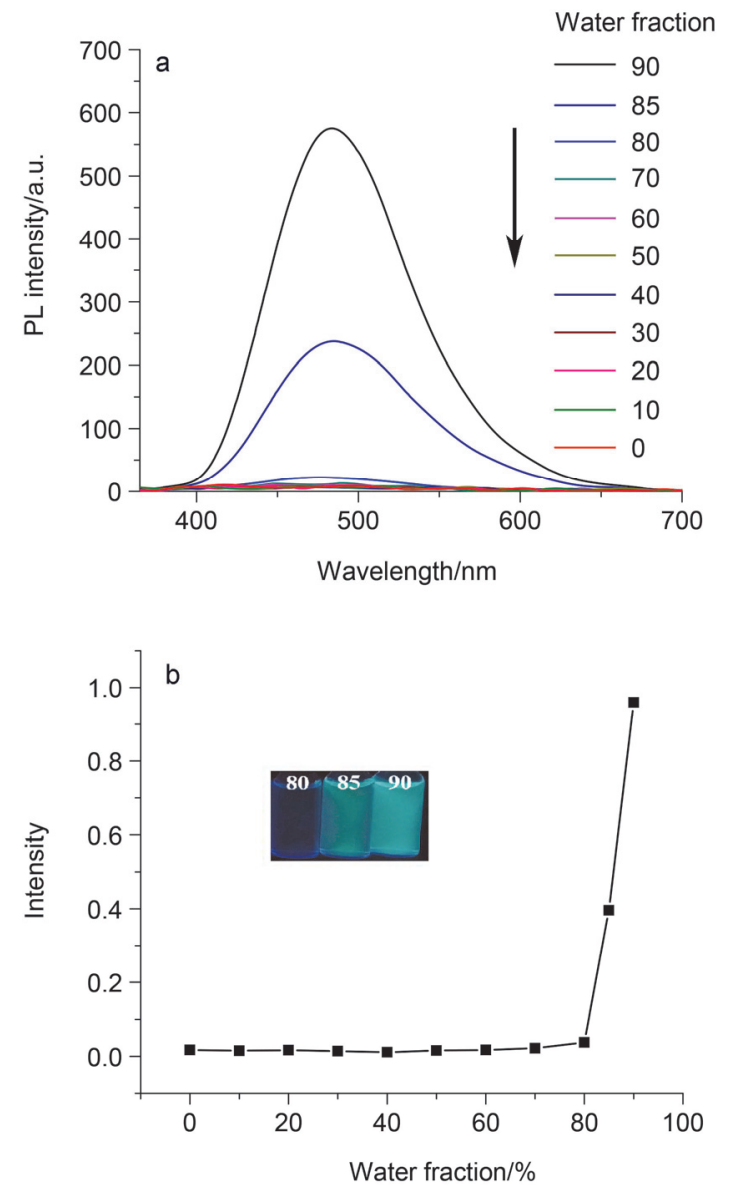

图 3 (a) BTBQ 在水含量不同的四氢呋喃/水混合溶液中的苂光发射 光谱. (b)发光强度与水含量的关系曲线图, BTBQ 的浓度: $1.0 \times 10^{-5}$ $\mathrm{mol} \cdot \mathrm{L}^{-1}$, 激发波长: $400 \mathrm{~nm}$. 插图: BTBQ 分别在水含量为 $80 \%, 85 \%$ 和 $90 \%$ 的四氢呋喃/水混合溶液中在 $365 \mathrm{~nm}$ 紫外光照射下的照片

Figure 3 (a) Fluorescence emission spectra of BTBQ in the THF/water with different water fractions $\left(f_{\mathrm{w}}\right)$. (b) Plot of PL intensity vs water fraction. Concentration: $1.0 \times 10^{-5} \mathrm{~mol} \cdot \mathrm{L}^{-1}, \lambda_{\mathrm{ex}}=400 \mathrm{~nm}$. Inset: photos of BTBQ in THF/water with $f_{\mathrm{w}}$ of $80 \%, 85 \%$ and $90 \%$ under $365 \mathrm{~nm}$ illumination
子产率达到 0.60, BTPQ 和 DBTPQ 在固态下分别发射 较强的蓝色荧光和绿色荧光, 固态荧光量子产率分别为 0.16 和 0.56 . 可见, 所合成的四苯乙烯修饰的喹喔啉衍 生物可在固态发光材料(如，苂光传感材料等)中得到应 用.

\section{5 压致苂光变色行为}

如图 4a, 4c 所示, 合成得到的固体 BTPQ 和 DBTPQ 分别发射蓝色和蓝绿色荧光, 最大发射波长分 别位于 $466 \mathrm{~nm}$ 和 $491 \mathrm{~nm}$. 经研磨处理后, 它们的发射 波长分别红移至 $500 \mathrm{~nm}$ 和 $507 \mathrm{~nm}$, 发光颜色分别变成 蓝绿色和绿色, 二者均表现出明显的压致荧光变色行 为. 但是, 可以看出, 研磨所导致 BTPQ 和 DBTPQ 的 发射光谱红移程度不同, 前者红移了 $34 \mathrm{~nm}$, 后者红移 了 $16 \mathrm{~nm}$, 这可能是因为 2,3 -位苯环的存在使喹喔啉分 子 DBTPQ 在外力作用下趋于平面化的程度较低或堆积 方式改变的程度较低所致. 此外, 经研磨后, 固体 BTPQ 和 DBTPQ 的荧光量子产率分别增大到 0.30 和 0.62 , 这可能是由于在外力作用下分子趋于平面化, 其 共轭程度增大导致的. 研磨后的样品经二氯甲烷蒸气熏 蒸或热退火处理后, 它们的发光颜色可以恢复到研磨前 状态, 说明压致荧光变色过程是可逆的(图 S5, S6). 然 而, BTBQ 没有表现出压致荧光变色行为, 推测可能是 由于它不是典型的给受体型分子的缘故.

为了讨论分子的压致苂光变色机理，探讨了 BTPQ 和 DBTPQ 在不同固体状态下的 X-射线衍射(XRD)图 谱. 如图 $4 \mathrm{~b}, 4 \mathrm{~d}$ 所示, 合成得到的固体 BTPQ 和 DBTPQ 均给出多个较尖锐衍射峰, 表现出晶体态的特征, 分子 的有序排列程度较高; 经研磨后, 样品的衍射峰大都消 失, 只能观察到很微弱的信号, 说明研磨后样品成无定 形态; 研磨后的样品再经二氯甲烷蒸气熏蒸, 部分衍射 峰得以恢复, 说明样品由无定形态逐渐转化成结晶态. 所以, BTPQ 和 DBTPQ 在不同固态下发光颜色的改变 是由于分子堆积方式不同引起的. 该结论可以由化合物 在不同固态下的差示量热扫描(DSC)结果得到进一步证 实, 如图 S7 所示, 研磨后的样品 BTPQ 和 DBTPQ 在熔 点之前均出现一个明显的放热峰, 相转变温度分别为 178.0 和 $170.0{ }^{\circ} \mathrm{C}$, 推测该放热峰对应于热处理过程中 固体由无定型态向晶体态的转变 ${ }^{[17]}$. 另外, 从合成得到 的固体 BTPQ 的 DSC 曲线上可以观察到一个位于 $170{ }^{\circ} \mathrm{C}$ 左右的吸热峰, 为探讨对应于该峰的相变过程, 测试了合成样品在 $170{ }^{\circ} \mathrm{C}$ 热退火处理前后的 XRD 图谱 (图 S8), 发现二者的衍射峰明显不同, 说明固体 BTPQ 在 $170{ }^{\circ} \mathrm{C}$ 左右发生了不同晶型之间的转变.

\section{6 酸响应}

由于喹喔啉单元的质子化会引起其吸收光谱和苂 光光谱的改变 ${ }^{[14]}$, 因此, 测试了所合成的目标分子对酸 的传感性能. 当将三氟乙酸(TFA)滴加到 BTBQ 的二氯 

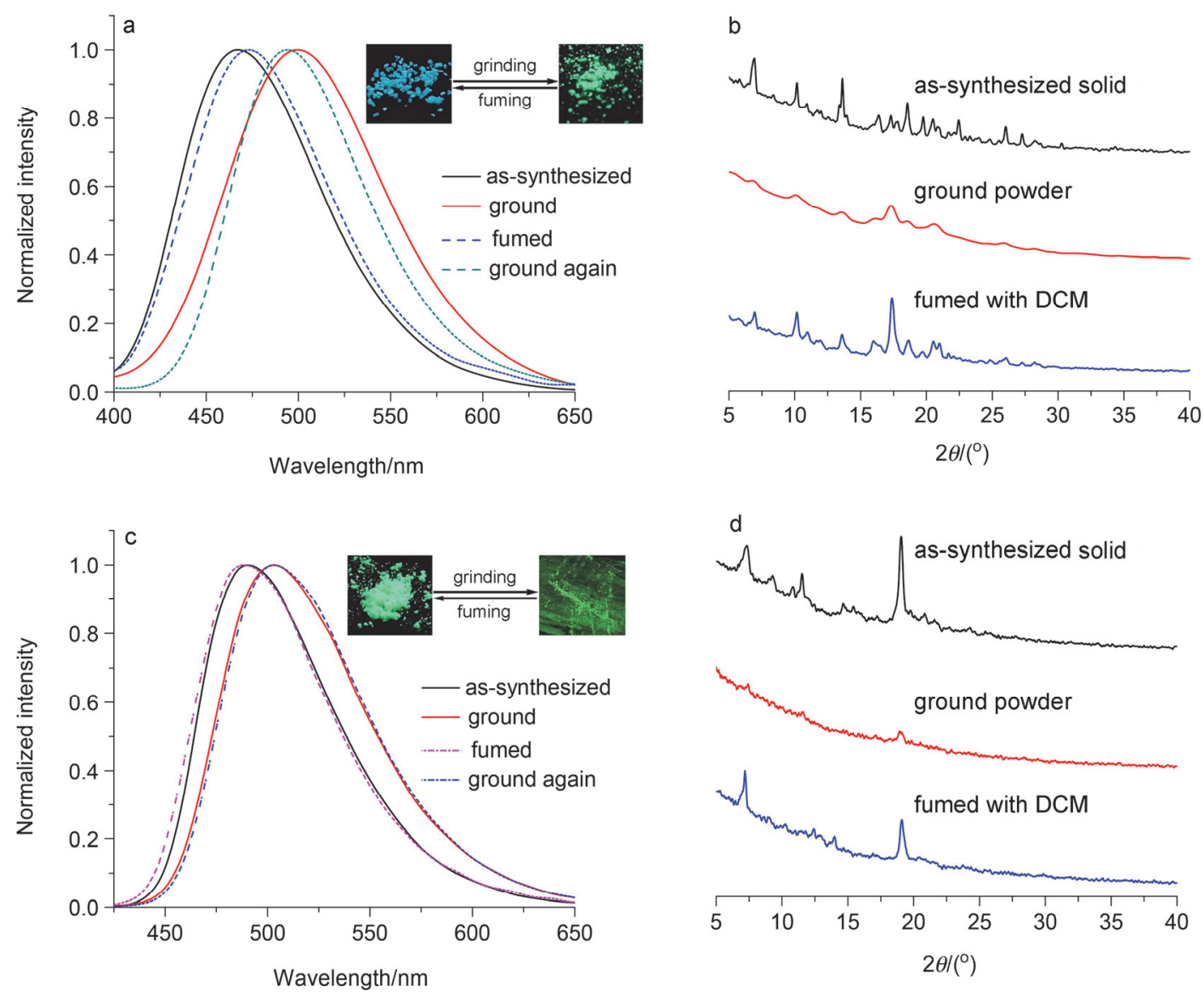

图 4 归一化的 BTPQ (a)和 DBTPQ (c)在不同固态下的苂光发射光谱(激发波长: $400 \mathrm{~nm}$ ); BTPQ (b)和 DBTPQ (d)在不同固态下的粉末 X-射线衍 射图. 插图: BTPQ 和 DBTPQ 在压致荧光变色过程中的照片

Figure 4 Normalized fluorescence emission spectra of BTPQ (a) and DBTPQ (c) in different solid states excited at $400 \mathrm{~nm}$; XRD patterns of BTPQ (b) and DBTPQ (d) in different solid states. Inset: photos of BTPQ and DBTPQ during piezofluorochromic processes

甲烷溶液中, 发现无色的溶液逐渐变为红色(图 S9b), 且溶液的蓝色荧光被显著猝灭(图 S10b). 从紫外-可见 吸收光谱滴定实验中可以看出, 在 BTBQ 的二氯甲烷溶 液中, 随着 TFA 含量的增大, 位于 $240 \sim 400 \mathrm{~nm}$ 的吸收 带逐渐下降, 且在 $450 \sim 500 \mathrm{~nm}$ 之间出现一个新吸收峰 (图 S9), 说明 TFA 与 BTBQ 作用后形成了一个新的物 种, 即质子化的喹咥啉衍生物. 如图 S10 所示, 随着 TFA 含量的增加, BTBQ 在二氯甲烷溶液中的荧光发射 强度逐渐下降, 因此, BTBQ 可作为传感材料用于检测 酸. 另外, BTPQ 和 DBTPQ 的苂光发射光谱不能在酸诱 导下发生改变, 可能是由于在喹喔啉 5,8 -位上连有体积 较大的四苯乙烯单元, 空阻效应阻碍了酸分子的进攻, 使 BTPQ 和 DBTPQ 不能被质子化造成的.

由于 BTBQ 具有 AIE 性质, 其固体有较强的发光能 力, 故考察了固体 BTBQ 对气态 TFA 的传感性能. 如图 5 所示, 在 TFA 蒸气熏蒸下, 淡黄色固体 BTBQ 变成红 色, 且位于 $500 \mathrm{~nm}$ 的最大发射峰在短时间内被完全猝 灭, 蓝绿色荧光消失, 可能是由于质子化 BTBQ 发生了 光诱导电子转移引起的. 此外, 如果将被 TFA 熏蒸后的 不发光的固体样品 BTBQ 暴露在三乙胺(TEA)饱和蒸气
中, 位于 $500 \mathrm{~nm}$ 的苂光强度几乎可以完全恢复, 且这个 过程可以重复多次, 说明 BTBQ 对 TFA 和 TEA 气体具 有可逆的苂光响应．同时也考察了固体 BTBQ 对乙酸、 浓盐酸和浓硝酸蒸气的苂光传感性能(图 S11, S12, S13), 发现其对上述酸均有一定程度的荧光响应. 为探讨 BTBQ 固体对酸响应的选择性, 将其暴露在 DMF、甲 苯、石油醚、乙酸乙酯、环己烷、乙醇、乙腈和丙酮蒸 气中, 发现其苂光强度几乎没有发生变化(图 S14), 说 明 BTBQ 对酸蒸气的响应具有选择性. 因此, BTBQ 可 作为传感材料用于酸蒸气的可视化检测.

\section{3 结论}

本文合成了双四苯乙烯修饰的喹喔啉衍生物. 研究 结果表明, 所合成的目标分子均具有 AIE 活性，当四苯 乙烯单元位于喹喔啉的 2,3-位时, 分子 BTBQ 给出最显 著的 AIE 性质, 其固态荧光量子产率可达 0.60. 尽管机 械外力不能引起其固态苂光光谱的变化, 但是, 酸蒸气 能快速猝灭其苂光, 同时固体由淡黄色变为红色, 此外, 固体的吸收和发光光谱能在 TEA 蒸气作用下得以恢复, 

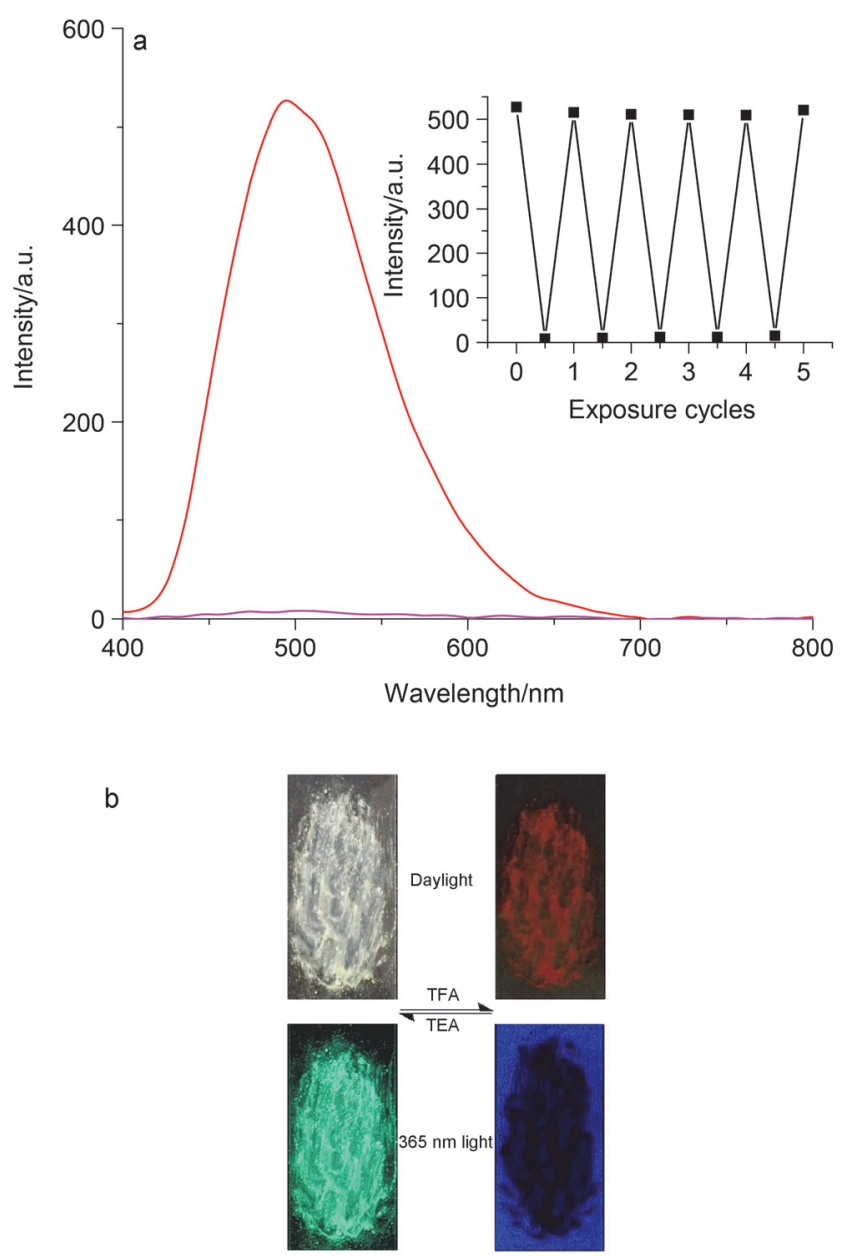

图 5 (a) BTBQ 在三氟乙酸饱和蒸气熏蒸前后的苂光发射光谱. 插 图: BTBQ 固体在 $500 \mathrm{~nm}$ 处的苂光发射强度在饱和三氟乙酸和三乙胺 蒸气循环熏蒸下的可逆变化图. (b)在日光和 $365 \mathrm{~nm}$ 紫外光照射下的 固体 BTBQ 在三氟乙酸和三乙胺蒸气循环熏蒸下的照片

Figure 5 (a) Fluorescence emission spectra of BTBQ upon exposed to saturated TFA vapor. Inset: Reversible fluorescence emission intensity at $500 \mathrm{~nm}$ for BTBQ solid upon exposed to saturated vapors of TEA and TFA; (b) Photos of BTBQ solid before and after exposed to TEA and TFA vapors under daylight and $365 \mathrm{~nm}$ light.

且该过程具有可逆性. 故, BTBQ 可作为传感材料用于 酸蒸气的可视化检测. 对于 BTPQ 和 DBTPQ 而言, 由 于四苯乙烯单元修饰在喹喔啉的 5,8-位, 空间效应使喹 喔啉难以被酸进攻, 因此, 二者对酸没有响应能力. 但 是, BTPQ 和 DBTPQ 表现出明显的压致荧光变色行为, 外力作用下的结晶态和无定形态之间的转化是引起材 料在不同固态下发光颜色不同的原因, 材料可望在防 伪、应力传感等方面得到应用. 本工作可为具有预期功 能的刺激响应性荧光染料的分子设计提供思路.

\section{4 实验部分}

5,8-双-(4-三苯基乙烯基-苯基)-喹喔啉(BTPQ)：将 $1.2 \mathrm{~g}(3.3 \mathrm{mmol})$ 化合物 $\mathbf{1} 、 2.5 \mathrm{~g}(6.6 \mathrm{mmol})$ 化合物 2 和
$4.5 \mathrm{~g}(33 \mathrm{mmol}) \mathrm{K}_{2} \mathrm{CO}_{3}$ 置于含有 $80 \mathrm{~mL}$ 甲苯和 $20 \mathrm{~mL}$ 乙 醇的干燥三口瓶中，体系在真空条件下鼓吹氮气 10 min, 然后向体系中加入 $0.01 \mathrm{~g}(0.87 \mathrm{mmol}) \mathrm{Pd}\left(\mathrm{PPh}_{3}\right)_{4}$ 并 继续鼓吹氮气 $10 \mathrm{~min}$. 体系回流 $24 \mathrm{~h}$, 静置冷却至室温, 倒入 $400 \mathrm{~mL}$ 蒸馏水中, 分离有机相, 饱和食盐水洗涤, 无水硫酸镁干燥，旋干溶剂. 经柱层析提纯(石油醚/二 氯甲烷 $=1 / 1)$, 得蓝绿色固体 $1.95 \mathrm{~g}$. 产率为 $75 \%$. m.p. $305 \sim 332{ }^{\circ} \mathrm{C} .{ }^{1} \mathrm{H}$ NMR $\left(400 \mathrm{MHz}, \mathrm{CDCl}_{3}\right) \delta: 8.83(\mathrm{~s}, 2 \mathrm{H})$, $7.79(\mathrm{~s}, 2 \mathrm{H}), 7.48(\mathrm{~d}, J=8.4 \mathrm{~Hz}, 4 \mathrm{H}), 7.08 \sim 7.16(\mathrm{~m}$, $30 \mathrm{H}$ ), 7.05 (dt, $J=5.0,2.7 \mathrm{~Hz}, 4 \mathrm{H}$ ) (图 S15); ${ }^{13} \mathrm{C} \mathrm{NMR}$ $\left(101 \mathrm{MHz}, \mathrm{CDCl}_{3}\right) \delta: 143.92,143.90,143.78,142.88$, $141.31,141.08,140.72,139.79,136.34,131.57,131.39$, $131.37,131.09,130.21,129.93,127.78,127.68,127.62$, 126.51, 126.46, 126.40 (图 S16). FT-IR (KBr) v: 698, 754, $817,864,918,980,1028,1074,1184,1263,1384,1442$, 1491, 1596, 2955, $3024 \mathrm{~cm}^{-1}$. MALDI-TOF MS: $m / z$ : calcd for $\mathrm{C}_{60} \mathrm{H}_{42} \mathrm{~N}_{2}$ : 790.33; found $791.5[\mathrm{M}+\mathrm{H}]^{+}$(图 S17).

2,3-二苯基-5,8-双-(4-三苯基乙烯基-苯基)喹喔啉 (DBTPQ)：以 3 和 2 为原料，用合成 BTPQ 相似的方法 制备 DBTPQ. 得亮绿色固体. 产率 80\%. m.p. 275 $292{ }^{\circ} \mathrm{C} .{ }^{1} \mathrm{H}$ NMR $\left(400 \mathrm{MHz}, \mathrm{CDCl}_{3}\right) \delta: 7.83(\mathrm{~s}, 2 \mathrm{H}), 7.64$ $(\mathrm{d}, J=8.3 \mathrm{~Hz}, 4 \mathrm{H}), 7.58(\mathrm{~d}, J=7.0 \mathrm{~Hz}, 4 \mathrm{H}), 7.35$ (dt, $J=$ $22.1,7.1 \mathrm{~Hz}, 8 \mathrm{H}), 7.14 \sim 7.20$ (m, 28H), $7.11(\mathrm{~d}, J=2.7$ $\mathrm{Hz}, 4 \mathrm{H})$ (图 S18); ${ }^{13} \mathrm{C}$ NMR (101 MHz, $\left.\mathrm{CDCl}_{3}\right) \delta: 150.77$, $144.00,143.92,143.84,143.08,142.90,141.16,140.78$, $139.09,138.88,138.37,136.32,131.65,131.49,131.45$, $131.01,130.18,130.09,129.70,128.82,128.18,127.81$, 127.68, 126.58, 126.50, 126.43 (图 S19). FT-IR (KBr) v: 696, 754, 987, 1246, 1385, 1458, 1490, 1560, 1654, 1772, $2364 \mathrm{~cm}^{-1}$. MALDI-TOF MS: $\mathrm{m} / z$ : calcd for $\mathrm{C}_{72} \mathrm{H}_{50} \mathrm{~N}_{2}$ : 942.4; found $943.5[\mathrm{M}+\mathrm{H}]^{+}$(图 S20).

1,2-双-(4'-三苯基乙烯基二苯基-4-基)-乙烷-1,2-二 酮(5): 以 4 和 2 为原料, 用合成 BTPQ 相似的方法制备 5. 得黄色固体. 产率 $63 \%$. m.p.: $200 \sim 210{ }^{\circ} \mathrm{C}$. ${ }^{1} \mathrm{H}$ NMR $\left(400 \mathrm{MHz}, \mathrm{CDCl}_{3}\right) \delta: 8.04(\mathrm{~d}, J=8.1 \mathrm{~Hz}, 4 \mathrm{H}), 7.71(\mathrm{~d}, J=$ $8.1 \mathrm{~Hz}, 4 \mathrm{H}), 7.41(\mathrm{~d}, J=8.0 \mathrm{~Hz}, 4 \mathrm{H}), 7.14(\mathrm{dd}, J=9.1,4.6$ $\mathrm{Hz}, 22 \mathrm{H}$ ), 7.08 (dt, $J=13.6,3.8 \mathrm{~Hz}, 12 \mathrm{H}$ ) (图 S21). ${ }^{13} \mathrm{C}$ NMR $\left(101 \mathrm{MHz}, \mathrm{CDCl}_{3}\right) \delta: 194.11,147.07,144.42$, $143.56,143.54,143.50,141.77,140.17,137.09,132.07$, $131.62,131.39,131.34,131.32,130.48,127.86,127.81$, $127.70,127.33,126.70,126.64,126.60,126.55$ (图 S22). FT-IR (KBr) v: 695, 755, 818, 856, 1004, 1172, 1221, 1443, 1491, 1598, 1673, 2320, 2599, $3026 \mathrm{~cm}^{-1}$. MALDITOF MS: $m / z$ : calcd for $\mathrm{C}_{66} \mathrm{H}_{46} \mathrm{O}_{2}: 870.4$; found 869.9 (图 S23).

2,3-双-(4'-三苯基乙烯基-二苯基-4-基)-喹喔啉 (BTBQ): 将 $0.1 \mathrm{~g}(0.92 \mathrm{mmol})$ 邻苯二胺和 $0.81 \mathrm{~g}(0.92$ 
$\mathrm{mmol}$ ) 化合物 5 溶于 $50 \mathrm{~mL}$ 乙酸溶液中. 体系回流过夜. 待反应完毕, 体系冷却至室温, 倒入 $400 \mathrm{~mL}$ 蒸馏水中, 收集有机相, 饱和食盐水洗涤, 无水硫酸镁干燥, 旋干 溶剂. 经柱层析提纯 (石油醚/二氯甲烷 $=3 / 1$ ), 得浅绿色 固体 0.82 g. 产率为 $95 \%$. m.p. $310 \sim 330{ }^{\circ} \mathrm{C} .{ }^{1} \mathrm{H}$ NMR $\left(400 \mathrm{MHz}, \mathrm{CDCl}_{3}\right) \delta: 7.17 \sim 8.21(\mathrm{~m}, 2 \mathrm{H}), 7.76 \sim 7.80(\mathrm{~m}$, $2 \mathrm{H}), 7.58(\mathrm{dd}, J=24.3,8.4 \mathrm{~Hz}, 8 \mathrm{H}), 7.38(\mathrm{~d}, J=8.4 \mathrm{~Hz}$, $4 \mathrm{H}), 7.03 \sim 7.17(\mathrm{~m}, 34 \mathrm{H})$ (图 S24). ${ }^{13} \mathrm{C} \mathrm{NMR}(101 \mathrm{MHz}$, $\left.\mathrm{CDCl}_{3}\right) \delta: 153.04,143.76,143.74,143.72,143.26,141.31$, $141.25,141.05,140.47,137.99,137.90,131.90,131.45$, $131.38,130.30,129.99,129.21,127.84,127.75,127.68$, $126.76,126.58,126.55,126.50,126.23$ (图 S25). FT-IR (KBr) v: 628, 698, 763, 819, 977, 1004, 1058, 1220, 1344, 1394, 1492, 1600, 2923, $3025 \mathrm{~cm}^{-1}$. MALDI-TOF MS: $m / z$ : calcd for $\mathrm{C}_{72} \mathrm{H}_{50} \mathrm{~N}_{2}$ : 942.4; found $943.6[\mathrm{M}+\mathrm{H}]^{+}$(图 S26).

\section{References}

[1] (a) Sagara, Y.; Kato, T. Nat. Chem. 2009, 1, 605; (b) Chi, Z.; Zhang, X.; Xu, B.; Zhou, X.; Ma, C.; Zhang, Y.; Liu, S.; Xu, J. J. Chem. Soc. Rev. 2012, 41, 3878; (c) Yao, X. D.; Chi, Z. G. Sci. China Chem. 2013，43，1090. (姚献东, 池振国, 中国科学, 2013，43, 1090.)

[2] Luo, J.; Xie, Z.; Lam, J. W. Y.; Cheng, L.; Chen, H.; Qiu, C.; Kwok, H. S.; Zhan, X.; Liu, Y.; Zhu, D.; Tang, B. Z. Chem. Commun. 2001, 1740 .

[3] (a) Hong, Y.; Lam, J. W. Y.; Tang, B. Z. Chem. Soc. Rev. 2011, 40, 5361; (b) Zhao, Z. J.; Lam, J. W. Y.; Tang, B. Z. Soft Matter 2013, 9 , 4564 .

[4] (a) Peng, B. Y.; Xu, S. D.; Chi, Z. G.; Zhang, X. Q.; Zhang, Y.; Xu, J. R. Prog. Chem. 2003, 25, 1085. (彭邦银, 许适当, 池振国, 张锡 奇, 张艺, 许家瑞, 化学进展, 2003, 25, 1085); (b) Huang, T.; Wang, Z. Y.; Qin, A. J.; Sun, J. Z.; Tang, B. Z. Acta Chim. Sinica 2013, 71, 979. (黄田, 汪昭昒, 秦安军, 孙景志, 唐本忠, 化学学 报, 2013, 71, 979.)

[5] (a) Misra, R.; Jadhav, T.; Dhokale, B.; Mobin, S. M. Chem. Commun. 2014, 50, 9076; (b) Luo, X.; Li, J.; Li, C.; Heng, L.; Dong, Y.; Liu, Z.; Bo, Z.; Tang, B. Adv Mater. 2011, 23, 3261; (c) Wang, J.; Mei, J.; Hu, R.; Sun, J.; Qin, A.; Tang, B. J. Am. Chem. Soc. 2012, 134, 9956; (d) Li, H.; Chi, Z.; Xu, B.; Zhang, X.; Li, X.; Liu, S.; Zhang, Y.; Xu, J. J. Mater. Chem. 2011, 21, 3760; (e) Zhou, X.; Li, H.; Chi, Z.; Zhang, X.; Zhang, J.; Xu, B.; Zhang, Y.; Liu, S.; Xu, J. New J. Chem. 2012, 36, 685.

[6] (a) Wang, Y.; Liu, W.; Bu, L.; Li, J.; Zheng, M.; Zhang, D.; Sun, M.; Tao, Y.; Xue, S.; Yang, W. J. Mater. Chem. C 2013, 1, 856; (b) Yagai, S.; Seki, T.; Kitamura, T.; Würthner, F. Angew. Chem., Int. Ed. 2008, 47, 3367; (c) Dong, Y.; Xu, B.; Zhang, J.; Tan, X.; Wang, L.; Chen, J.; Lv, H.; Wen, S.; Li, B.; Ye, L.; Zou, B.; Tian, W. J. Angew. Chem. Int. Ed. 2012, 51, 10782.
[7] Gong, Y.; Tan, Y.; Liu, J.; Lu, P.; Feng, C.; Yuan, W.; Lu, Y.; Sun, J.; He, G.; Zhang, Y. Chem. Commun. 2013, 49, 4009.

[8] (a) An, B. K.; Kwon, S. K.; Jung, S. D.; Park, S. Y. J. Am. Chem. Soc. 2002, 124, 14410; (b) Yuan, W. Z.; Tan, Y.; Gong, Y.; Lu, P.; Lam, J. W. Y.; Shen, X.; Feng, C.; Sung, H. H. Y.; Lu, Y.; Williams, I. D.; Sun, J.; Zhang, Y.; Tang, B. Adv. Mater. 2013, 25, 2837; (c) Gong, Y.; Liu, J.; Zhang, Y.; He, G.; Lu, Y.; Fan, W. B.; Yuan, W. Z.; Sun, J. Z.; Zhang, Y. J. Mater. Chem. C 2014, $2,7552$.

[9] (a) Sagara, Y.; Mutai, T.; Yoshikawa, I.; Araki, K. J. Am. Chem. Soc. 2007, 129, 1520; (b) Yamane, S.; Sagara, Y.; Mutai, T.; Araki, K.; Kato, T. J. Mater. Chem. C 2013, 1, 2648.

[10] (a) Shen, X. Y.; Yuan, W. Z.; Liu, Y.; Zhao, Q.; Lu, P.; Ma, Y.; Williams, I. D.; Qin, A.; Sun, J. Z.; Tang, B. Z. J. Phys. Chem. C 2012, 116, 10541; (b) Shen, X. Y.; Wang, Y. J.; Zhao, E.; Yuan, W. Z.; Liu, Y.; Lu, P.; Qin, A.; Ma, Y.; Sun, J. Z.; Tang, B. Z. J. Phys. Chem. C 2013, 117, 7334; (c) Kim, F. S.; Guo, X.; Watson, M. D.; Jenekhe, S A. Adv. Mater. 2010, 22, 478; (e) Lin, T. C.; He, G.; Zheng, S. Q. J. Mater. Chem. 2006, 16, 2490

[11] (a) Ooyama, Y.; Ito, G.; Fukuoka, H.; Nagano, T.; Kagawa, Y.; Imae, I.; Komaguchi, K.; Harima, Y. Tetrahedron 2010, 66, 7268; (b) Ooyama, Y.; Harima, Y. J. Mater. Chem. 2011, 21, 8372.

[12] (a) Xue, P. C.; Yao, B. Q.; Sun, J. B.; Xu, Q. X.; Chen, P.; Zhang, Z. Q.; Lu, R. J. Mater. Chem. C 2014, 2, 3942; (b) Xue, P. C.; Chen, P.; Jia, J. H.; Xu, Q. X.; Sun, J. B.; Yao, B. Q.; Zhang, Z. Q.; Lu, R. Chem. Commun. 2014, 50, 2569; (c) Zhang, Z. Q.; Xue, P. C.; Gong, P.; Zhang, G. H.; Peng, J.; Lu, R. J. Mater. Chem. C 2014, 2, 9543 ; (d) Xue, P. C.; Yao, B. Q.; Wang, P. P.; Sun, J. B.; Zhang, Z. Q.; Lu, R. RSC Adv. 2014, 4, 58732.

[13] (a) Xue, P. C.; Yao, B. Q.; Liu, X. H.; Sun, J. B.; Gong, P.; Zhang, Z. Q.; Qian, C.; Zhang, Y.; Lu, R. J. Mater. Chem. C 2015, 3, 1018; (b) Zhang, Z. Q.; Wu, Z.; Sun, J. B.; Yao, B. Q.; Zhang, G. H.; Xue, P. C.; Lu, R. J. Mater. Chem. C 2015, 3, 4921; (c) Zhang, G. H.; Sun, J. B.; Xue, P. C.; Zhang, Z. Q.; Gong, P.; Peng, J.; Lu, R. J. Mater. Chem. C 2015, 3, 2925.

[14] Muniz, F. M.; Alcazar, V.; Sanz, F.; Simon, L.; de Arriba, A. L. F.; Raposo, C.; Moran, J. R. Eur. J. Org. Chem. 2010, 6179.

[15] Frisch, M. J.; Trucks, G. W.; Schlegel, H. B.; Scuseria, G. E.; Robb, M. A.; Cheeseman, J. R.; Scalmani, G.; Barone, V.; Mennucci, B.; Petersson, G. A.; Nakatsuji, H.; Caricato, M.; Li, X.; Hratchian, H. P.; Izmaylov, A.; Bloino, F. J.; Zheng, G.; Sonnenberg, J. L.; Hada, M.; Ehara, M.; Toyota, K.; Fukuda, R.; Hasegawa, J.; Ishida, M.; Nakajima, T.; Honda, Y.; Kitao, O.; Nakai, H.; Vreven, T.; Montgomery Jr, J. A.; Peralta, J. E.; Ogliaro, F.; Bearpark, M.; Heyd, J. J.; Brothers, E.; Kudin, K. N.; Staroverov, V. N.; Kobayashi, R.; Normand, J.; Raghavachari, K.; Rendell, A.; Burant, J. C.; Iyengar, S. S.; Tomasi, J. M.; Rega, N.; Millam, J. M.; Klene, M.; Knox, J. E.; Cross, J. B.; Bakken, V.; Adamo, C.; Jaramillo, J.; Gomperts, R.; Stratmann, R. E.; Yazyev, O.; Austin, A. J.; Cammi, R.; Pomelli, C.; Ochterski, J. W.; Martin, R. L.; Morokuma, K.; Zakrzewski, V. G.; Voth, G. A.; Salvador, P.; Dannenberg, J. J.; Dapprich, S.; Daniels, A. D.; Farkas, O.; Foresman, J. B.; Ortiz, J. V.; Cioslowski, J.; Fox, D. J. Gaussian 09, Revision A. 02, Gaussian, Inc., Wallingford, CT, 2009.

[16] Chi, Z. G.; He, K. Q.; Li, H. Y.; Zhang, X. Q.; Xu, B. J.; Liu, S. W.; Zhang, Y.; Xu, J. R. Chem. J. Chin. Univ. 2012, 33, 725. (池振国, 何克强，李海银，张锡奇，许炳佳，刘四委，张艺，许家瑞，高等 学校化学学报, 2012, 33, 725.)

[17] Vasanthan, N.; Manne, J.; Krishnama, A. Ind. Eng. Chem. Res. 2013, $52,17920$. 